\title{
Order allocation in a multiple suppliers-manufacturers environment within a dynamic cluster
}

\author{
Paolo Renna $^{1}$ - Giovanni Perrone ${ }^{2}$
}

Received: 30 July 2014 / Accepted: 6 March 2015 / Published online: 21 March 2015

(C) Springer-Verlag London 2015

\begin{abstract}
This study faces the problem of how to evaluate order allocation strategies in a multiple suppliersmanufacturers environment within a dynamic industry cluster that is an environment in which suppliers adopt a policy based on their performance to select the clusters in which to operate. Therefore, the allocation strategy performed by the manufacturers is crucial to keep a long-term supply chain partnership with a specific industry cluster. We use simulation to evaluate order allocation strategies under different market conditions. We show how an allocation strategy that takes into account the whole supply chain's perspective leads to a sustainable development of the clusters of suppliers.
\end{abstract}

Keywords Network enterprises · Coordination · Order allocation $\cdot$ Cluster $\cdot$ Simulation

\section{Introduction}

It is quite acknowledged how long-term partnership between manufacturers and supplier can improve competitiveness of Original Equipment Manufacturers (OEMs) [1]. It is also quite common that long-term suppliers are usually organised in industry clusters (i.e., steel industry [2] and US Petrochemical firms [3]).

Lavie [4] finds an increase from 32 to $95 \%$ for the percentage of corporations in the US software industry that

Paolo Renna

paolo.renna@unibas.it

Giovanni Perrone

giovanni.perrone@unipa.it

1 University of Basilicata, Potenza, Italy

2 University of Palermo, Palermo, Italy engaged in alliances between the beginning and the end of the 1990s.

Information and Communication Technologies can help in improving cooperation among clustered suppliers, making in this way the OEMs further more competitive $[5,6]$. Several concepts of network enterprises have been discussed in literature: extended enterprises, virtual enterprises, cluster concept, and production networks. The term 'extended enterprises' was introduced by Browne et al. [7] as an interenterprises network able to respond to the new pressures (reduced product life cycles, time-based competition, etc.). The virtual enterprises are defined as a network of independent companies managed through information technology and characterised by the temporary nature of relationships [8]. An extension of virtual enterprise is the cluster concept that comprises a heterarchical network of companies, their customers, and the suppliers of everything that is needed for running such a network [6].

One of the crucial issues in an industry cluster is the way the OEM allocates the orders to the cluster; indeed, the order allocation procedure might help to keep the cluster compact and more cooperating. This is the reason why several scholars, in production literature, have faced the order allocation issue. However, few researches have focused on the stability of a cluster due to the OEM's allocation policy.

Ulbrich et al. [9] presented a study of three networked organizations in Austria and Switzerland identifying six success factors: partners with complementary competencies that meet task requirements; open and transparent communication; high level of commitment; similarity regarding authority and rank within the firm; negotiation and agreement on rules of cooperation; and reasonable expectation of success. The order allocation is crucial for the high level of commitment and reasonable expectation of success in terms of revenue of the suppliers. 
The research proposed in this paper concerns the order allocation within industry cluster composed by several suppliers that provide an OEM. The main contributions of this paper are (a) an order allocation strategy able to balance the production of the suppliers and (b) a decision-making procedure allowing the suppliers to decide whether to participate or leave the OEM's cluster. A simulation environment has been developed to test how the order allocation strategy combined with the cluster dynamicity can improve the performance of the whole supply chain.

The rest of the paper is structured as it follows: Section 2 presents an overview of the literature on the order allocation in industry clusters. Section 3 describes the research context and the order allocation policies tested. The decision support models for the dynamic industry cluster are presented in Section 4. In Sections 5 and 6, we respectively present the simulation test environment and simulation results. Finally, conclusions and further research paths are drawn in Section 7.

\section{Literature review}

Several researches have been proposed on order allocation strategies and suppliers selection, but few papers discussed the evolution of a network with the dynamic change of the partners related to the order allocation strategy.

Kawtummachai and Hop [10] investigated the problem of allocating products to suppliers at operational level and try to minimize purchasing cost based on service satisfaction history. They argued that very few researchers have tried the optimization approach to order allocation.

Yang et al. [11] underlined how multi-plant order allocation problem is apparently an integer programming, solving such NP-hard problem usually takes a great deal of time as the dimension increases. Although the genetic algorithm demonstrates its feasibility and efficiency in solving this integer programming, more research is needed to further reduce the calculation time.

Chituc and Nof [12] proposed three dimensional performance analysis approach to determine join/leave/remain decisions in collaborative network organisations based on general attributes of agility, cost, and profitability. Jiang et al. [13] highlighted as the key attributes of a dynamic strategic alliance are the complementarity of partners' resources.

Some recent contributes concerning the allocation of orders to be manufactured face the problem as a capacity allocation/ sharing decision in production networks.

The capacity-sharing problem in a network of independent plants is analyzed under a cooperative game approach [14] and collaborative demand protocols [15]; however, the composition of the network in the aforementioned works is considered static.

Scholz-Reiter et al. [16] also addressed the problem of capacity allocation within a production network. They modeled the dynamic production network as a multi-class queuing network and investigate the robustness and stability of the network as dependent on the customer demand and production processes.

Yoon and Nof [17] developed affiliation/dissociation protocols in a collaborative network of enterprises when a firm, or a set of firms, needs to evaluate the anticipated reward of the collaboration network. They proposed a centralised approach with information sharing.

Haleh and Hamidi [18] proposed a fuzzy multi-objective linear programming model to support order allocation decisions between conflicting tangible and intangible factors. Much of the information in this part of supply chain management remains vague, which plays to the strengths of a fuzzy method.

Xiang et al. [19] proposed a load-equilibrium allocation order policy. The authors, in this case, take into account the past orders delay to evaluate a more realistic state of the capacity available. However, the clusters are static and the policy does not consider the whole cluster performance.

Renna [20] analysed the dynamic network problem in case of independent plants. In his work, the author proposed a methodology to make the decision to participate or leave a network on the basis of the local knowledge without sharing of information; in order to accomplish that, the author proposed a capacity sharing model based on a negotiation approach.

Chen [21] investigated the coordination mechanism in a supply chain consisting of one manufacturer and multiple competing suppliers in the electronic market. The author analysed two general price-only policies, i.e., the wholesale price policy and the catalogue policy, both of which are based on the reverse Vickrey auction [22].

Villa and Bruno [23] discussed how the European Commission is stimulating research on what could be the antidote of the crisis: the development of profitable SME aggregations in terms of either poles of competitiveness, network of competence, clusters, or industrial districts.

Thus, as the reader can notice, order allocation policy has not been associated with the decision of a supplier to participate to the cluster or not. Therefore, our contribution to the literature is to fill this gap. Thus, in our model, suppliers can dynamically decide whether to participate or leaving the cluster. We will show how, by designing a proper allocation strategy that takes into account the whole cluster's perspective, possible to make the performance better off in a long-term consideration. In order to do that, we propose an allocation strategy based on the load-equilibrium among the suppliers of a cluster. Moreover, the suppliers may decide whether to participate or leave the cluster using a periodic review policy. A classical capacity-based allocation strategy is used as a benchmark for our model (as some works used the same benchmark; e.g., see [19]). This is also motivated by the dynamic evolution of the clusters' members, the approaches proposed in literature concern static clusters. 


\section{Research context}

The research context concerns a set of manufacturers, i.e., an industry cluster that signed long-term contract with multiple suppliers. Thus, a stable cooperation between the OEM and its industry cluster leads to a full sharing of the order information without delay. Let us assume that the total planning horizon is divided into $N$ sub-periods $(t=1, \ldots, N)$; at the beginning of each sub-period, the OEM collects the demand and allocates order to the suppliers of its industry cluster (see Fig. 1).

Figure 1 shows the research context; each OEM faces with its market demand. Each OEM established long-term contracts with multiple suppliers included in a cluster. Each OEM allocates the production orders of its market using a production allocation strategy.

Moreover, the clusters are dynamic; when the contracts between the generic OEM and its suppliers need to be renewed, a dynamic cluster strategy is activated. In particular, the OEM evaluates if the number of suppliers have to be increased/decreased or can be stable. The options of the suppliers are to continue in the cluster, sign a contract only with another OEM, or sign contracts with multiple OEMs. The strategies of OEMs and suppliers depend on the performance obtained by the previous collaboration (mainly the production allocation strategy).

The parameters and variables used in this paper are expressed as it follows.

\section{Industrial environment}

$i=1 \ldots, I \quad$ set of suppliers

$j=1, . ., J \quad$ set of OEMs

$t=1, . ., T$ planning period

$A_{j} \quad$ set of supplier $i$ within the cluster of the OEM $j$

$A_{i} \quad$ set of the OEMs that have a contract

with the supplier $i$

und $_{j} \quad$ a set of under-utilised suppliers in cluster $j$

$T_{p} \quad$ number of periods of the periodic review

of the dynamic policy

\section{OEMs}

$D_{t j} \quad$ demand of the OEM $j$ at period $t$

$P_{t i j} \quad$ production order allocation of the $\mathrm{OEM} j$ for the supplier $i$ at period $t$

$P_{t i j}^{I} \quad$ production order allocation of the $\operatorname{OEM} j$ for the under-utilisation supplier $i$ at period $t$

$P_{t i j}^{I I} \quad$ production order allocation of the OEM $j$ for the over-utilisation supplier $i$ at period $t$

$r e s D_{t j} \quad$ residual demand to allocate of the $\operatorname{OEM} j$ after the production allocation to the under-utilisation suppliers at period $t$

\section{Suppliers}

$C_{i} \quad$ capacity of the supplier $i$

$\mathrm{Cav}_{t i} \quad$ capacity available of the supplier $i$ at period $t$ after the OEM production allocation

Fig. 1 Research context

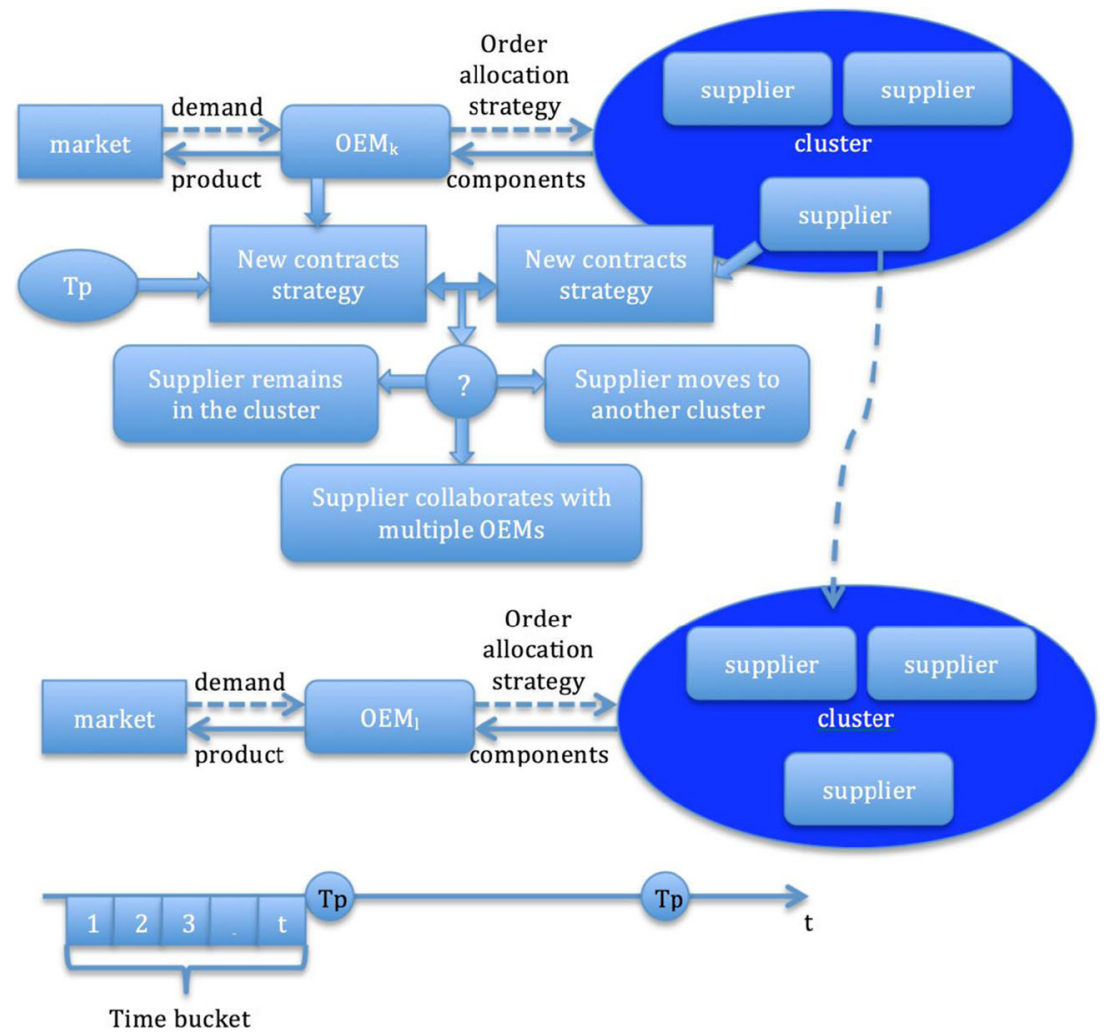


$S_{t i} \quad$ the production order quantity supplied in delay by the supplier $i$ at period $t$

$O_{t i} \quad$ is the production orders outside any clusters' participation (e.g., occasional orders)

$W L_{t i} \quad$ the total workload of the supplier $i$ at period $t$

$U T_{i}\left(T_{p}\right)$ the average utilization of the supplier $i$ over the last period

$U T a v_{t i}$ the average utilization of the supplier $i$ in the time bucket (last $T_{p}$ periods) until time $t$

$U T a v_{t j}$ the average utilization of the cluster $j$ in the time bucket (last $T_{p}$ periods) until time $t$

The first activity concerns the order allocation problem. The order allocation strategy determines the production load $P_{t i j}$ of the suppliers within the cluster at the beginning of each production period through the steps described in Section 2 (benchmark and proposed policy). Afterwards, each supplier $i$ computes the workload $W L_{t i}$ as it follows:

$W L_{t i}=\sum_{j \in A_{i}} P_{t i j}+O_{t i}+S_{t-1, i}$

where the workload due to the delayed production orders is computed as in (2):

$S_{t i}=\operatorname{MAX}\left[W L_{t i}-C_{i} ; 0\right]$, for $t \geq 1 ; S_{0 i}=0$

The average utilization of the supplier $i$ over the last $T p$ periods is computed as it follows:

$U T_{i}\left(T_{p}\right)=\frac{\sum_{t \in T p} W L_{t i}}{C_{i} \cdot T_{p}}$

\subsection{Capacity-based allocation strategy}

The amount of order to allocate to each supplier of an industry cluster is determined considering the capacity of each supplier of the cluster. The suppliers that cooperate in a stable cluster are characterized by the same price and quality; therefore, the capacity is the unique characteristic of each supplier. The allocation of the production order is determined as shown in (4):

$P_{t i j}=\frac{C_{i}}{\sum_{i \in A_{j}} C_{i}} \cdot D_{t j}$

Expression (4) defines the production allocation of each supplier $i$ at a specific period $t$ considering the individual capacity of the supplier $i$ related to the total capacity of the OEM's cluster $j$.
This strategy is widely adopted by most of the OEMs, but it is characterized by some limits. The main limit is related to the capacity of the supplier that does not reflect the real-time state of the supplier, but a static view. In particular, this strategy does not take into account the workload due to production orders in delay. This strategy is used as a benchmark of the proposed allocation strategy, which is discussed in the next sub-section.

\subsection{Utilization-based allocation strategy}

The proposed allocation strategy considers the utilization of the supplier's capacity over a determined periodic review in order to uniform the utilization of the suppliers within an industry cluster. The main objective is to assure an adequate level of satisfaction to all suppliers of the cluster in terms of production capacity allocated.

This strategy allocates the production orders in two steps. The first step concerns the computation of the average utilization of the suppliers within an industry cluster. The average utilization is computed within the time bucket considered. Let us consider a generic time bucket $t=1, . ., T p$ and let's indicate with $t_{\text {now }}$ the actual period in which the allocation process is activated $\left(t_{\text {now }}\right.$ is within the interval $\left.1, \ldots, T p\right)$. Expression 5 computes the average utilization of the periods before $t_{\text {now }}$ within the time bucket for each industry cluster. If $t_{\text {now }}$ is the first period of the time bucket, then the average utilization is zero. In this last case, the orders allocation follows the benchmark strategy described in Section 2.

$\operatorname{UTav}_{\text {tnow }, j}=\frac{\sum_{i \in A j} \sum_{t=1}^{t \text { now-1 }} \frac{W L_{t i}}{C_{i}}}{\operatorname{card}\left(A_{j}\right)}$, if $\mathrm{t}_{\text {now }}>1$;

else $U T a v_{\text {tnow }, j}=0$

where $\operatorname{card}\left(A_{j}\right)$ is the cardinality of set $A_{j}$.

Then, each supplier $i$ computes its average utilization as shown in expression (6):

$U \operatorname{Tav}_{\text {tnow }, i}=\frac{\sum_{j \in A i} \sum_{t=1}^{t \text { now- } 1} P_{t i j}}{C_{i} \cdot(\text { tnow-1) }}$, if $\mathrm{t}_{\text {now }}>1$;

else $U T a v_{\text {tnow }, i}=0$

The comparison between the average utilization of the generic supplier $i$ and the average utilization of the cluster allows to determine a set of under-utilised suppliers in each cluster $j\left(u n d_{j}\right)$. Thus, the suppliers of the set $u n d_{j}$ are the suppliers with higher priority for the production orders allocation. 
Two cases can be considered. In the first case, the suppliers of $u n d_{j}$ set are able to satisfy all the production capacity required by the $\mathrm{OEM}_{j}$. In this case, the production order is allocated only to the set of suppliers $u n d_{j}$ through the following expression:

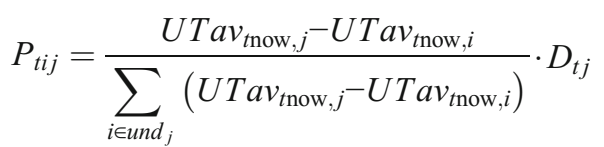

As the reader can notice, production orders are allocated in order to uniform the average utilization of the suppliers in each cluster.

In the second case, the set of $u n d_{j}$ suppliers is not able to satisfy all the production capacity required. In this case, the production order is allocated to the set of $u n d_{j}$ suppliers in order to reach the average utilization of the cluster (see expression 8).

$P_{t i j}{ }^{I}=\left(U \operatorname{Tav}_{\text {tnow }, j}-U \operatorname{Tav}_{\text {tnow }, i}\right) \cdot C_{i}, \forall i \in$ und $_{j}$

However, in this case, the OEM has still a residual demand $\left(r e s D_{t j}\right)$, computed according to (9), to allocate:

$r e s D_{t j}=D_{t j}-\sum_{i \in u n \mathrm{~d}_{j}} P_{t i j}{ }^{I}$

Residual demand is allocated through the expression (10):

$P_{t i j}{ }^{I I}=\frac{\operatorname{Cav}_{i}}{\sum_{i \in A_{j}} \operatorname{Cav}_{i}} \cdot \operatorname{res} D_{t j}$

where $\mathrm{Cav}_{i}$ is the capacity still available after having allocated orders to each under-utilised supplier $\left(u n d_{j}\right)$ according to (8); thus, $\mathrm{Cav}_{i}$ is equal to:

$\operatorname{Cav}_{t i}=C_{t i}-P_{t i j}{ }^{I}, \forall i \in u n d_{j}$

while, $\operatorname{Cav}_{t i}=C_{i}, \forall i \notin u n d_{j}$

Then, the production allocation is the sum of the two steps described above as shown in expression (13):

$P_{t i j}=P_{t i j}{ }^{I}+P_{t i j}{ }^{I I}$

This approach allows balancing the order allocated to the cluster's supplier in each period of the review policy. Thus, supplier utilization is not pre-determined while the cluster is more adaptable to the different market conditions.

\section{Dynamic cluster}

This research investigates how the production allocation strategy can affect the decisions of OEMs and suppliers when the contracts among them should be revised.
Dynamic cluster means that suppliers and OEMs can decide to modify the participation in the clusters. Thus, a periodic review strategy is undertaken by the parts in order to decide whether the contracts among suppliers and OEMs should be modified, changing in this way the composition of the OEM's cluster. The length of the periodic review depends on the contract signed $\left(T_{p}\right)$.

The methodology proposed is based on the evaluation on the performance measures obtained by the past production allocation orders.

Each supplier evaluates the following performance measures:

- average utilization over the last periodic review (see Eq. (3));

- workload fluctuations over the last periodic review. The fluctuation is computed as the ratio between the standard deviation and average of the workload.

From the point of view of the OEMs, the following performance measures are evaluated:

- average cluster utilization over the last periodic review;

- demand fluctuations over the last periodic review. The fluctuation is computed as the ratio between the standard deviation and the average of the market demand.

At each review period $\left(T_{p}\right)$, all OEMs and suppliers compute the above performance measures to decide the clusters' composition.

The OEMs and suppliers apply a set of rules (reported in Table 1) to decide among three possible strategies: increase, stable, and decrease.

As reported in Table 1, there are nine combinations of OEMs and suppliers' strategies.

The following combinations lead to change the clusters' composition:

- Combination A-Generally, there are two sets, OEMs and suppliers, that apply the 'increase' decision. The couple OEM-supplier with the higher difference between the unsatisfied demand (OEM) and average utilization (supplier) is established. The new couples of OEM and supplier are established until one of the two sets OEM with increase and supplier with increase strategy is empty. This strategy allows increasing the utilization of the suppliers satisfying the OEMs with higher unsatisfied demand.

- Combinations C, F, and I-Generally, in these cases, there is a sets of suppliers that apply the 'decrease' decision. The generic supplier of this set identifies the OEM that has lower importance in terms of workload allocated in past periods. The supplier can leave this OEM; if the 
Table 1 Suppliers and OEMs decision strategies

\begin{tabular}{|c|c|c|c|c|}
\hline \multicolumn{5}{|c|}{ Suppliers' decision strategies } \\
\hline \multicolumn{2}{|c|}{ Utilization } & \multicolumn{2}{|c|}{ Workload fluctuation } & Decision \\
\hline \multicolumn{2}{|l|}{ Low } & \multicolumn{2}{|c|}{ (Low, medium, and high) } & Increase \\
\hline \multicolumn{2}{|l|}{ High } & \multicolumn{2}{|c|}{ (Low, medium, and high) } & Decrease \\
\hline \multicolumn{2}{|l|}{ Medium } & \multicolumn{2}{|l|}{ High } & Decrease \\
\hline \multicolumn{4}{|c|}{ Other combinations } & Stable \\
\hline \multicolumn{5}{|c|}{ OEMs' decision strategies } \\
\hline \multicolumn{2}{|c|}{ Cluster's utilization } & \multicolumn{2}{|c|}{ Demand fluctuation } & Decision \\
\hline \multicolumn{2}{|l|}{ High } & \multicolumn{2}{|c|}{ (Low, medium, high) } & Increase \\
\hline \multicolumn{2}{|l|}{ Medium } & \multicolumn{2}{|l|}{ High } & Increase \\
\hline \multicolumn{2}{|l|}{ Low } & \multicolumn{2}{|l|}{ Low } & Decrease \\
\hline \multicolumn{4}{|c|}{ Other combinations } & Stable \\
\hline \multicolumn{5}{|c|}{ Strategies combination } \\
\hline \multicolumn{2}{|l|}{ Suppliers } & Increase & Stable & Decrease \\
\hline \multirow[t]{3}{*}{ OEMs } & Increase & A & B & $\mathrm{C}$ \\
\hline & Stable & $\mathrm{D}$ & $\mathrm{E}$ & $\mathrm{F}$ \\
\hline & Decrease & G & $\mathrm{H}$ & I \\
\hline
\end{tabular}

following two conditions are verified, the supplier works at least in two clusters; other suppliers compose the cluster that the supplier would leave. These conditions avoid the dissolution of a cluster or a supplier without participation in a cluster.

The clusters don't change the composition in other combinations (B, D, E, G, H). In particular, the combination $\mathrm{G}$ is characterized by opposite strategy of OEMs and suppliers. The other combinations (B, D, E, H) concern stable decisions.

\section{Simulation environment}

A simulation environment based on a JAVA package has been developed to test the proposed approach. The modeling formalism adopted here is a collection of independent objects interacting via messages. In particular, each object represents an agent and the system evolves through a message-sending engine managed by a discrete event scheduler. We have developed the following objects: the cluster mediator, the OEM, the supplier, and the statistical analysis. The mediator object is in charge of the objects' interaction with the coordination of the messages exchanged among the involved objects. Moreover, it is in charge with the system evolving by managing the discrete events of the simulation engine. The OEM object represents the manufacture; it has all the information, algorithms, and function for its implementation. The supplier object represents the supplier; it has all the information, algorithms, and function for its implementation. Finally, the statistical agent collects the simulative data in order to write the report of the simulation. Three OEMs with their industry cluster compose the environment investigated in the case study. Three suppliers that supply the same products compose each cluster. The length of the simulation is 24 periods.

Furthermore, because of the parameters extracted by the statistical distributions, and in order to guarantee a statistical validity of the results, for each run, the number of executed replications guarantees, for the output performance measures, that the length of confidence intervals ( $95 \%$ level) of the mean among replications is lower than $5 \%$ of the mean itself. The following performance measures have been considered to compare the strategies:

- average utilization of all suppliers $(u t)$;

- standard deviation of the suppliers' utilization ( $(\mathrm{dev})$;

- average production orders in delay (uns) of the suppliers;

- average capacity available for each industry cluster;

- average participant in each industry cluster; this index is the ratio between the average participation of the suppliers in the clusters over the time simulated and the initial number of suppliers in each cluster.

- standard deviation of the suppliers' capacity utilization.

The approaches proposed are simulated in four conditions: The benchmark condition characterised by capacity-based allocation and static cluster composition (C1); utilization-based allocation and static cluster (C2); capacity-based allocation and dynamic cluster (C3); and utilization-based allocation and dynamic cluster (C4). The value of the $T p$ is fixed to 3 periods. The suppliers and OEMs evaluate the conditions reported in the Table 1 as range values as shown in Table 2.

\section{Numerical results}

The numerical results obtained by the simulation experiments allow us to evaluate the benefit of the proposed approach.

The simulations have been conducted on the following cases:

- Case 1: capacity allocation policy and static cluster (benchmark);

- Case 2: utilization allocation policy and static cluster;

- Case 3: capacity allocation policy and dynamic cluster;

- Case 4: utilization allocation policy and dynamic cluster;

Table 2 Range values

\begin{tabular}{llll}
\hline & Low & Medium & High \\
\hline Fluctuations & $(0-0.3)$ & $(0.3-0.5)$ & $(0.75-1)$ \\
Utilization & $(0-0.6)$ & $(0.6-0.8)$ & $(0.8-1)$ \\
\hline
\end{tabular}


Table 3 Initial cluster composition and OEM's demand

\begin{tabular}{lllllr}
\hline OEM's composition & OEM & Suppliers & & & Cluster Capacity \\
Configuration 1 & Cluster 1 & Supplier 1 & Supplier 2 & Supplier 3 & 300 \\
& Cluster 2 & Supplier 4 & Supplier 5 & Supplier 6 & 300 \\
& Cluster 3 & Supplier 7 & Supplier 8 & Supplier 9 & 300 \\
& Capacity & 100 & 150 & 50 & 300 \\
Configuration 2 & Cluster 1 & Supplier 1 & Supplier 2 & Supplier 3 & 300 \\
& Cluster 2 & Supplier 4 & Supplier 5 & Supplier 6 & 300 \\
& Cluster 3 & Supplier 7 & Supplier 8 & Supplier 9 & 300 \\
Configuration 3 & Capacity & 100 & 100 & 100 & 300 \\
& Cluster 1 & Supplier 1,10 & Supplier 2,11 & Supplier 3,12 & 300 \\
Cluster 2 & Supplier 4,13 & Supplier 5,14 & Supplier 6,15 & Supplier 9,18 \\
OEMs' demand & Cluster 3 & Supplier 7,16 & Supplier 8,17 & 50 & \\
Capacity & 50 & 50 & OEM3 & \\
Demand 1 & & OEM1 & OEM2 & UNIF[100;500] & \\
Demand 2 & & UNIF[100;500] & UNIF[100;500] & \\
Demand 3 & & UNIF[150;250] & UNIF[150;250] & UNIF[100;500] & UNIF[100;500] \\
\hline
\end{tabular}

- Case 5: capacity allocation policy, dynamic cluster, and each supplier can operate within one cluster;

- Case 6: utilization allocation policy, dynamic cluster, and each supplier can operate within one cluster.

Table 3 reports the initial composition of the industry cluster with a capacity also reported in for each supplier. We consider three identical clusters without occasional orders $O_{t i}$ outside the cluster in order to evaluate only the policies proposed. Three configurations are considered with the same capacity of each cluster. The objective is to investigate how the capacity of the suppliers is relevant for the proposed policies.
Three cases have been considered for the OEMs' demand (see Table 3). Case 1 considers a demand equal for all OEMs with high fluctuations; case 2 considers two clusters underutilised at medium level and another over-utilised. Finally, case 3 considers two clusters under-utilised at lower level and two under-utilised.

The simulation results are reported in terms of percentage difference compared to the benchmark case (static cluster and capacity allocation strategy).

The first analysis conducted regards the value added by the proposed allocation strategy in the three possible comparisons: static, dynamic cluster $(d y n)$, and dynamic cluster when the suppliers can operate in only one cluster ( $d y n$ excl). Figure 2 reports the numerical results for the demand profile
Fig. 2 Unsatisfied demandallocation strategy—Demand profile 1

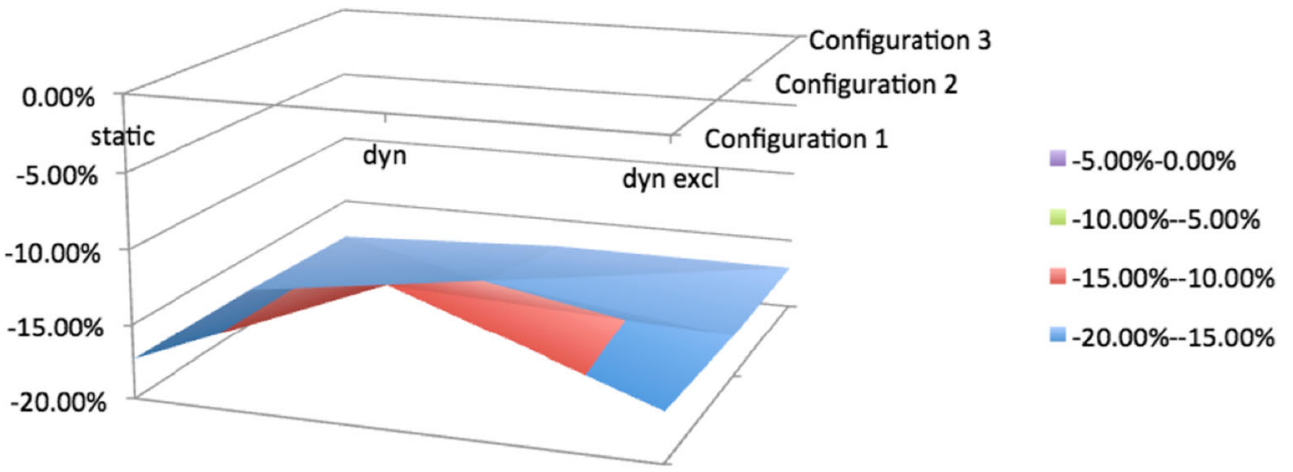

\begin{tabular}{|c|c|c|c|}
\hline & static & dyn & dyn excl \\
\hline Configuration 1 & $-17.35 \%$ & $-10.62 \%$ & $-16.69 \%$ \\
\hline Configuration 2 & $-17.45 \%$ & $-15.05 \%$ & $-17.15 \%$ \\
\hline Configuration 3 & $-17.87 \%$ & $-17.05 \%$ & $-17.08 \%$ \\
\hline
\end{tabular}


Fig. 3 Unsatisfied demandallocation strategy—Demand profile 2

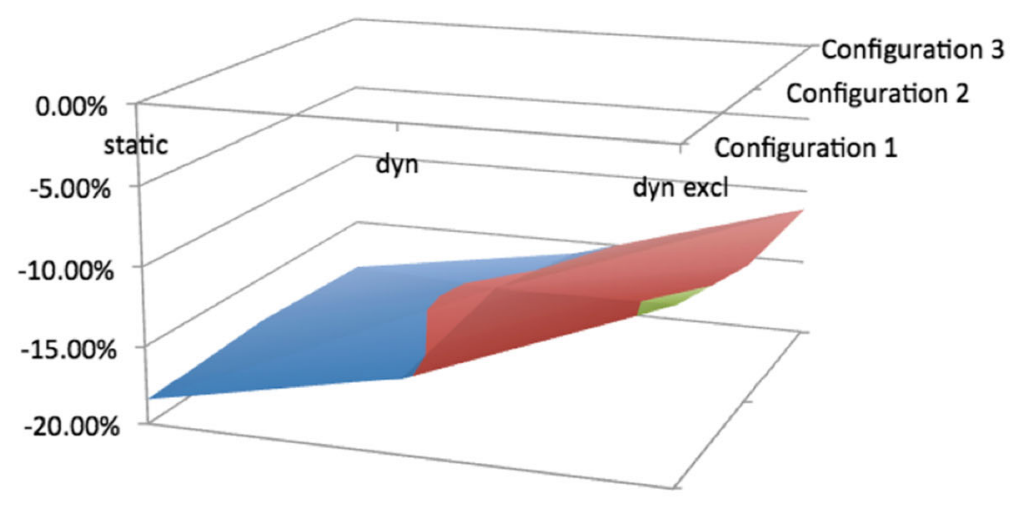

$-5.00 \%-0.00 \%$

$-10.00 \%--5.00 \%$

$=-15.00 \%-10.00 \%$

$-20.00 \%-15.00 \%$

\begin{tabular}{|l|c|c|c|}
\hline & static & dyn & dyn excl \\
\hline Configuration 1 & $-18.44 \%$ & $-15.29 \%$ & $-9.10 \%$ \\
\hline Configuration 2 & $-17.95 \%$ & $-14.11 \%$ & $-11.06 \%$ \\
\hline Configuration 3 & $-18.40 \%$ & $-15.89 \%$ & $-11.24 \%$ \\
\hline
\end{tabular}

1 in terms of reduction of unsatisfied demand and the trend of this performance.

Figure 2 shows that the proposed allocation strategy leads always to better results in terms of unsatisfied demand. The configurations of the suppliers' capacity have a low influence on this performance. Moreover, this demand profile (equal for all OEMs) is characterized by lower benefit in case of dynamic cluster and the configuration of the cluster composed by suppliers with different capacity (configuration 1). In case of demand similar among the clusters, the configurations ( 2 and 3 ) with clusters composed by suppliers with equal capacity lead to better results.

Figure 3 reports the same results for the demand profile 2 and shows the trend of this performance.

In this demand profile, the dynamic cluster with the suppliers that can operate in a single cluster leads always to worst results. The configurations of the suppliers' capacity have a low influence on the unsatisfied demand.

Figure 4 reports the same results for the demand profile 3 and shows the trend of this performance.

In this demand profile, the benefit of the proposed allocation strategy is higher in case of static cluster or dynamic cluster (with suppliers shared among the clusters) for the configuration 1 (suppliers with different capacity). In all other cases, the benefits are lower; in particular, in case of dynamic cluster when the suppliers can participate in one cluster.

The second analysis conducted regards the value added by the dynamic cluster approach. In this case, the comparisons are four: capacity allocation strategy (Cap), the utilization allocation strategy (Ut), capacity allocation strategy (Cap) and suppliers that operate in only one cluster (Cap excl), and the
Fig. 4 Unsatisfied demandallocation strategy_-Demand profile 3

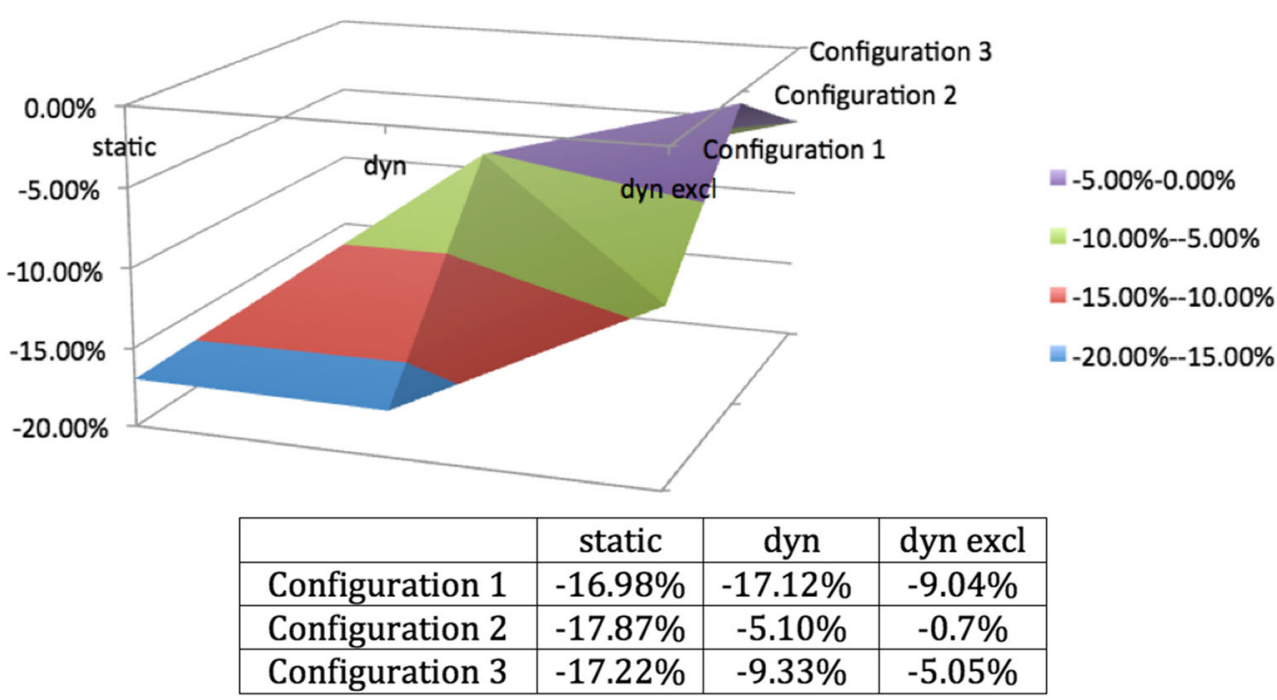


Fig. 5 Unsatisfied demanddynamic cluster-Demand profile 1

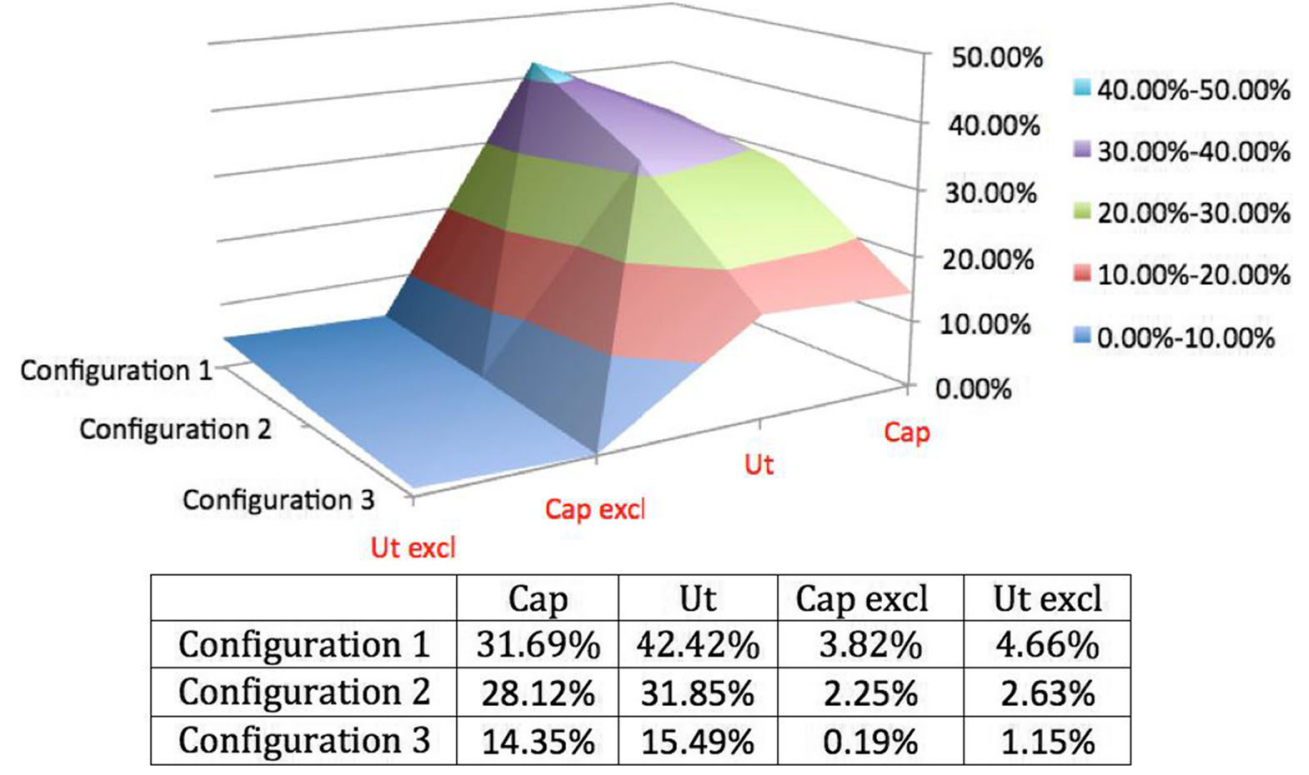

utilization allocation strategy (Ut) and suppliers that operate in only one cluster (Ut excl).

Figure 5 reports the results for the demand profile 1 and shows the trend of this performance.

The dynamic cluster mechanisms lead always to the worst results when the demand is the same among the OEMs. If the suppliers can participate to only one cluster, the increment of unsatisfied demand is limited.

Figure 6 reports the results for the demand profile 2 and shows the trend of this performance.

In this case, the dynamic cluster mechanisms allow to improving the performance of unsatisfied demand. In particular, the configuration 1 when the suppliers have the same capacity and the number of suppliers is lower lead to better results.

Figure 7 reports the results for the demand profile 3 and shows the trend of this performance.
In this demand scenario, the reduction of unsatisfied demand is always relevant compared to the other demand scenarios. The configuration with lower benefit is the case when the suppliers have the same capacity and the number of suppliers is higher (lower capacity for each supplier).

From the point of view of suppliers, the reduction of the unsatisfied demand leads to increase the production. Therefore, the reduction of unsatisfied demand is an improvement of the profit for OEMs and suppliers.

However, the increment of production does not increase considerably the average utilization of all suppliers.

In literature, the standard deviation or coefficient of variation is considered an important index (e.g., see [24]) to improve the capacity utilization equity among suppliers. Table 4 reports the standard deviation of the capacity utilization of suppliers in simulated cases.
Fig. 6 Unsatisfied demanddynamic cluster-Demand profile 2

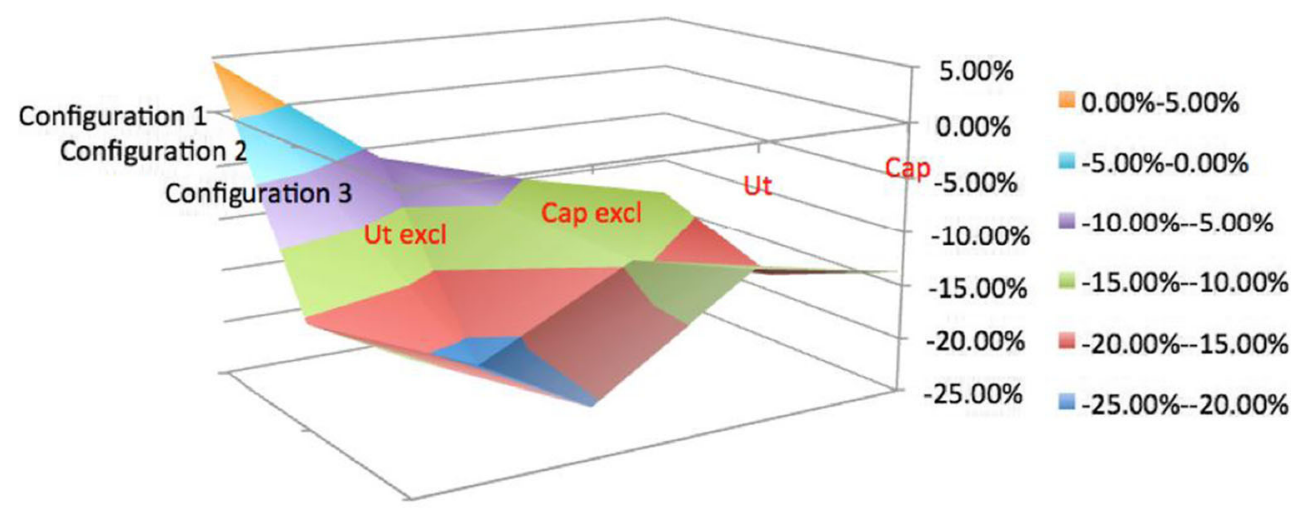

\begin{tabular}{|c|c|c|c|c|}
\hline & Cap & Ut & Cap excl & Ut excl \\
\hline Configuration 1 & $-13.49 \%$ & $-10.15 \%$ & $-6.03 \%$ & $4.72 \%$ \\
\hline Configuration 2 & $-18.34 \%$ & $-14.52 \%$ & $-22.00 \%$ & $-15.45 \%$ \\
\hline Configuration 3 & $-13.73 \%$ & $-11.08 \%$ & $-20.45 \%$ & $-13.48 \%$ \\
\hline
\end{tabular}


Fig. 7 Unsatisfied demanddynamic cluster-Demand profile 3

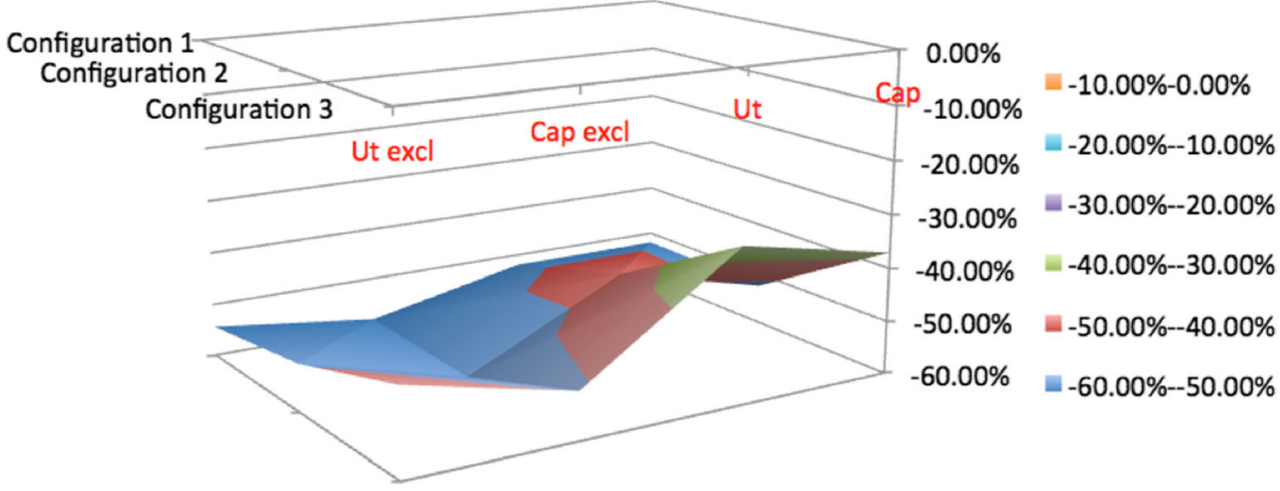

\begin{tabular}{|c|c|c|c|c|}
\hline & Cap & Ut & Cap excl & Ut excl \\
\hline Configuration 1 & $-52.01 \%$ & $-52.10 \%$ & $-58.33 \%$ & $-54.35 \%$ \\
\hline Configuration 2 & $-52.45 \%$ & $-45.08 \%$ & $-59.57 \%$ & $-51.12 \%$ \\
\hline Configuration 3 & $-37.05 \%$ & $-31.05 \%$ & $-51.13 \%$ & $-43.95 \%$ \\
\hline
\end{tabular}

The dynamic cluster approach reduces the standard deviation of the suppliers' utilization when the demand is different among the OEMs (demand profile 2 and 3). The production allocation approach does not affect this performance.

Therefore, the reduction of the unsatisfied demand is obtained by a better distribution of the capacity utilization with better capacity utilization equity among the suppliers.

The average participation of the suppliers in each cluster evaluates the stability of the cluster over the periods simulated.

For the demand 1 (the demand of the markets is the same for all OEMs), the clusters are very stable and this index is always about 1 (this means that the initial configuration of the cluster does not change).

Table 5 reports the index of the suppliers' participation in clusters for demand 2 and 3. The following considerations are discussed. The first consideration concerns the stability of the clusters; in all cases, the index has a value greater than 0.5 .

Table 4 Standard deviations of the suppliers' utilization

\begin{tabular}{llrrr}
\hline \multicolumn{1}{l}{ Cap } & Ut & Cap excl & \multicolumn{1}{l}{ Ut excl } \\
\hline Demand 1 & & & & \\
Configuration 1 & $160.00 \%$ & $83.33 \%$ & $440.00 \%$ & $500.00 \%$ \\
Configuration 2 & $160.00 \%$ & $83.33 \%$ & $440.00 \%$ & $500.00 \%$ \\
Configuration 3 & $600.00 \%$ & $0.00 \%$ & $1400.00 \%$ & $9985.71 \%$ \\
Demand 2 & & & & \\
Configuration 1 & $-31.25 \%$ & $-33.48 \%$ & $-34.05 \%$ & $-35.48 \%$ \\
Configuration 2 & $-28.64 \%$ & $-28.27 \%$ & $-33.36 \%$ & $-33.06 \%$ \\
Configuration 3 & $-28.64 \%$ & $-28.27 \%$ & $-33.36 \%$ & $-33.06 \%$ \\
Demand 3 & & & & \\
Configuration 1 & $-47.86 \%$ & $-37.95 \%$ & $-56.23 \%$ & $-59.27 \%$ \\
Configuration 2 & $-45.94 \%$ & $-45.06 \%$ & $-53.74 \%$ & $-52.76 \%$ \\
Configuration 3 & $-45.94 \%$ & $-45.06 \%$ & $-53.74 \%$ & $-52.76 \%$ \\
\hline
\end{tabular}

This means that the participants in each cluster are always over the $50 \%$ of the initial configuration. Therefore, the clusters keep a sustainable composition.

The second consideration regards the average values computed; these values highlight how the main effects are due to the market demand profile and the configuration of the suppliers, while the production allocation strategy has low influence on this index.

When the sum of the suppliers' participation (sum in Table 5) is greater than 3, it means that some suppliers are shared among the OEMs. As the reader can notice, the suppliers operate in more clusters when the number of suppliers is higher with lower capacity (configuration 3 ) for the demand 2. In case of demand 3, the number of suppliers shared among the clusters is higher, but in case of high number of suppliers with lower capacity the degree of cluster sharing decreases.

However, the degree of shared suppliers is characterized by lower values; therefore, the dynamic cluster approaches improve the performance with adequate level of shared suppliers among the clusters.

Obviously, in case of exclusive participation of the suppliers, the sum is always 3 .

Finally, it is reported the better case for each combination of demand scenario and configuration of suppliers.

Table 6 reports the better cases for the OEMs (reduction of unsatisfied demand). When the demand is similar among the OEMs, the proposed allocation strategy in static cluster (case 2 ) is the better case. When the demand is different among the OEMs, the dynamic cluster when the suppliers can participate in one cluster is the better case.

Table 6 reports the better case for the maximization of the suppliers' utilization.

As reported in Appendix, the difference among the cases tested is very low. However, for this performance, the 
Table 5 Suppliers' participation in the clusters

Configuration 1

\begin{tabular}{|c|c|c|c|c|c|c|c|c|c|c|}
\hline & $\begin{array}{l}\text { Demand } 2 \\
\text { Cap }\end{array}$ & $\mathrm{Ut}$ & Cap Excl & Ut Excl & Average & $\begin{array}{l}\text { Demand } \\
\text { Cap }\end{array}$ & $\mathrm{Ut}$ & Cap Excl & Ut Excl & Average \\
\hline Cluster 1 & 0.95 & 0.95 & 0.94 & 0.95 & 0.95 & 0.86 & 0.88 & 0.78 & 0.81 & 0.83 \\
\hline Cluster 2 & 1.02 & 1.00 & 0.94 & 0.95 & 0.98 & 1.00 & 1.00 & 0.79 & 0.81 & 0.90 \\
\hline Cluster 3 & 1.11 & 1.11 & 1.12 & 1.11 & 1.11 & 1.37 & 1.35 & 1.42 & 1.38 & 1.38 \\
\hline Sum & 3.08 & 3.07 & 3.00 & 3.00 & & 3.23 & 3.22 & 3.00 & 3.00 & \\
\hline \multicolumn{11}{|c|}{ Configuration 2} \\
\hline Cluster 1 & 0.95 & 0.95 & 0.94 & 0.94 & 0.97 & 0.86 & 0.86 & 0.78 & 0.79 & 0.82 \\
\hline Cluster 2 & 1.00 & 1.00 & 0.94 & 0.94 & 0.98 & 1.00 & 1.00 & 0.80 & 0.80 & 0.90 \\
\hline Cluster 3 & 1.11 & 1.11 & 1.12 & 1.12 & 1.07 & 1.38 & 1.38 & 1.42 & 1.42 & 1.40 \\
\hline Sum & 3.06 & 3.06 & 3.00 & 3.00 & & 3.24 & 3.23 & 3.00 & 3.00 & \\
\hline \multicolumn{11}{|c|}{ Configuration 3} \\
\hline Cluster 1 & 0.97 & 0.97 & 0.96 & 0.96 & 0.97 & 0.90 & 0.90 & 0.85 & 0.85 & 0.87 \\
\hline Cluster 2 & 1.00 & 1.00 & 0.97 & 0.97 & 0.98 & 1.00 & 1.00 & 0.86 & 0.86 & 0.93 \\
\hline Cluster 3 & 1.06 & 1.06 & 1.07 & 1.07 & 1.07 & 1.24 & 1.23 & 1.29 & 1.29 & 1.26 \\
\hline Sum & 3.24 & 3.23 & 3.00 & 3.00 & & 3.14 & 3.13 & 3.00 & 3.00 & \\
\hline Average & 1.02 & 1.02 & 1.00 & 1.00 & & 1.02 & 1.02 & 1.00 & 1.00 & \\
\hline
\end{tabular}

possibility of the suppliers to participate in more clusters leads to better results (case 5).

Table 6 reports the better cases in terms of production distribution among the suppliers. This performance is the deviation of the production allocation.

When the demand is different among the OEMs, the dynamic cluster when the suppliers can participate in one cluster is the better case (case 6). Only for the distribution of the production among the suppliers in case of similar demand among the OEMs, the capacity allocation strategy leads to better performance.

Table 6 Better cases for suppliers and OEMs

\begin{tabular}{llll}
\hline OEMs' performance & & & \\
& Demand 1 & Demand 2 & \multicolumn{1}{l}{$\begin{array}{l}\text { Demand 3 } \\
\text { Case 6 }\end{array}$} \\
Configuration 1 & Case 2 & Case 4 & Case 6 \\
Configuration 2 & Case 2 & Case 6 & Case 6 \\
Configuration 3 & Case 2 & Case 6 & \\
Suppliers' utilization & & & Demand 3 \\
& Demand 1 & Demand 2 & Case 1 \\
Configuration 1 & Case 1 & Case 1 & Case 5 \\
Configuration 2 & Case 5 & Case 5 & Case 6 \\
Configuration 3 & Case 5 & Case 5 & \\
Suppliers' utilization distribution & & Demand 3 \\
& Demand 1 & Demand 2 & Case 6 \\
Configuration 1 & Case 1 & Case 6 & Case 6 \\
Configuration 2 & Case 1 & Case 6 & Case 6 \\
Configuration 3 & Case 1 & Case 6 &
\end{tabular}

\section{Conclusions}

The research proposed in this paper deals with the multiple suppliers-manufactures problem within dynamic industry cluster. This paper proposes an allocation of the suppliers within a cluster based on the utilization evaluation. The proposed approach, when compared to a classical capacityallocation one, leads to better results in terms of reduction of the delay of the production order allocated. This benefit is obtained keeping the same level of other performance. The benefits are evaluated both in static and dynamic cluster, considering the OEMs' demand fluctuations and difference among the OEMs and the different compositions of the cluster in terms of suppliers' capacity.

When the clusters change dynamically, the improvement obtained with the proposed approach is lower. However, in the dynamic case, the proposed approach allows the suppliers to make a better decision about the clusters in which to be involved. Indeed, the possibility to modify the participation of the suppliers in the clusters allows improving the distribution of the workload among the suppliers with higher suppliers' satisfaction and lower production fluctuations. In case of longterm partnership, the production allocation is more important of costs and quality. The proposed production allocation method allows reducing the fluctuations and increasing the resource utilization of the suppliers. In these conditions, the OEM's cluster is more stable with mutual benefit and higher supply chain efficiency.

The dynamic cluster with the suppliers that participate in only one cluster leads to better results both for OEMs and suppliers in the great part of cases tested. From the point of 
view of suppliers' capacity composition, the better results are obtained when the suppliers have the same capacity with higher capacity for each supplier (lower fragmentation).

The simulation environment developed is a valid tool to support the decision on the strategy to use to obtain the better performance in the conditions in which operate the OEMs and suppliers.

Further research paths concern the decision of the suppliers to change cluster considering a more complex decision environment involving parameters such as cost, transport distance, etc. Moreover, the influence of $T_{p}$ on the performance measures will be investigated.

\section{References}

1. Wiendahl H-P, Lutz S (2002) Production in networks. CIRP Ann Manuf Technol 51(2):573-586

2. Sadler D (2004) Cluster evolution, the transformation of old industrial regions and the steel industry supply chain in North East England. Reg Stud 38(1):55-66

3. Patti AL (2006) Economic clusters and the supply chain: a case study. Supply Chain Manag Int J 11(3):266-270

4. Lavie D (2007) Alliance portfolios and firm performance: a study of value creation and appropriation in the U.S. software industry. Strateg Manag J 28(12):1187-1212

5. Sari B, Sen T, Kilic SE (2007) Formation of dynamic virtual enterprises and enterprise networks. Int J Adv Manuf Technol 34(1112):1246-1262

6. Porter M (1998) Clusters and the new economies of competition. Harv Bus Rev 76(6):77-90

7. Browne J, Sackett P, Wortmann H (1995) Industry requirements and associated research issues in the extended enterprise. In: Ladet $\mathrm{P}$, Vernadat $\mathrm{F}$ (eds) Integrated manufacturing systems engineering. Chapman \& Hall, London, pp 2-28

8. Byrne JA, Brandt R, Port O (1993) The virtual corporation. Bus Week 8:36-40

9. Ulbrich S, Troitzsch H, van den Anker F, Pluss A, Huber C (2011) How teams in networked organisations develop collaborative capability: processes, critical incidents and success factors. Production Planning \& Control 22(5-6):488-500

10. Kawtummachai R, Hop NV (2005) Order allocation in a multiple supplier environment. Int J Prod Econ 93-94:231-238

11. Yang FC, Chen KT, Wang MT, Chang PY, Sun KC (2010) Mathematical modelling of multi- plant order allocation problem and solving by genetic algorithm with matrix representation. Int $\mathrm{J}$ Adv Manuf Technol 51(9-12):1251-1259

12. Chituc C-M, Nof SY (2007) The Join/Leave/Remain (JLR) decision in collaborative networked organizations. Comput Ind Eng 53(1):173-195

13. Jiang X, Li Y, Gao S (2008) The stability of strategic alliances: characteristics, factors and stages. J Int Manag 14(2):173-189

14. Renna P, Argoneto P (2011) Capacity sharing in a network of independent factories: a cooperative game theory approach. Robot Comput Integr Manuf 27(2):405-417

15. Nof SY, Seok H (2014) Dynamic coalition reformation for adaptive demand and capacity sharing. Int J Prod Econ 147(Part A):136-146

16. Scholz-Reiter B, Wirth F, Makuschewitz T, Schönlein M (2011) Robust capacity allocation in dynamic production networks. CIRP Ann Manuf Technol 60(1):445-448

17. Yoon SW, Nof SY (2011) Affiliation/dissociation decision models in demand and capacity sharing collaborative network. Int J Prod Econ 130(2): 135-143

18. Haleh H, Hamidi A (2011) A fuzzy MCDM model for allocating orders to suppliers in a supply chain under uncertainty over a multiperiod time horizon. Expert Syst Appl 38(8):9076-9083

19. Xiang W, Song F, Ye F (2014) Order allocation for multiple supplydemand networks within a cluster. J Intell Manuf 25(6):1367-1376

20. Renna P (2013) Decision model to support the SMEs' decision to participate or leave a collaborative network. Int J Prod Res 51(7): 1973-1983

21. Chen K (2013) Procurement strategies and coordination mechanism of the supply chain with one manufacturer and multiple suppliers. Int J Prod Econ 138(1):125-135

22. Vickrey W (1961) Counterspeculation, auctions, and competitive sealed tenders. J Financ 16(1):8-37

23. Villa A, Bruno G (2013) Promoting SME cooperative aggregations: main criteria and contractual models. Int J Prod Res 51(23-24): 7439-7447

24. Chan FTS, Chung SH, Choy KL (2006) Optimization of order fulfillment in distribution network problems. J Intell Manuf 17(3): 307-319 